\title{
Microparticles Based on Low Melting Hydrophilic Polymers Prepared by Spray Drying
}

\author{
M. HOMAR, J. KERČ
}

Lek Pharmaceuticals,d.d., Sandoz Development Center Slovenia, Verovškova 57, 1526 Ljubljana, Slovenia E-mail: miha.homar@sandoz.com (M. Homar)

Sci Pharm. 2010; 78: 604

doi:10.3797/scipharm.cespt.8.PDD15

The enhancement of dissolution rate is one of the most commonly used approaches to improve the bioavailability of drugs, since for a great extent of new drug substances their absorption is limited with their dissolution rate. The aim of this work was to prepare and optimize microparticles containing ketoprofen using spray-drying technology, which would enhance the dissolution rate of ketoprofen, which is poorly soluble at lower $\mathrm{pH}$ values. Different hydrophilic polymers with relatively low melting point (polyethylene glycol 4000 and 6000 , poloxamer 188, Gelucire 44/14 and 50/13) were used to prepare microparticles by spray drying. Due to the low melting point of the polymers, spray drying parameters were carefully optimized to ensure reasonable yields. Of the process parameters, atomizing pressure was shown to be the most important factor influencing product yield. Product yield was also greatly impacted by the adhesion of the particles to the inside wall of the spray dryer due to the sticking of the carrier material. A positive correlation between the melting point of the polymers and the product yield was established - the lower the melting point of the polymer, the lower was the yield. Carriers with promising yield (polyethylene glycol 4000 and poloxamer 188 with the average yield of $34,4 \%$ and $43,0 \%$, respectively) were chosen for the preparation of the drug loaded microparticles. A reference sample was prepared using lactose as a carrier. In all cases ketoprofen was not dissolved in the final mixture and ketoprofen suspensions in carrier polymer solutions were spray dried. During the preparation of drug loaded microparticles, the drug concentration in the suspension was shown to have a great impact on the overall yield. Due to extremely poor yield when polyethylene glycol 4000 was used as a carrier, only samples prepared with poloxamer 188 and lactose were further studied. Image analysis was used to determine the size and shape of microparticles, which were recorded by scanning electron microscope (SEM). The dissolution rate of ketoprofen from the microparticles prepared with poloxamer 188 was enhanced compared to microparticles prepared with lactose as a carrier. All prepared microparticles exhibited markedly increased dissolution rate compared to ketoprofen alone. The differential scanning calorimetry (DSC) confirmed a similar thermal behavior of ketoprofen microparticles and corresponding physical mixtures which indicates the absence of chemical interaction between ketoprofen and water soluble carrier. It was also shown that there was no significant conversion of crystalline ketoprofen to the amorphous form during the spray drying process. 